\title{
EchoGéo
}

19 | 2012

Du littoral à la haute mer : quelles recherches récentes en géographie?

\section{Comment étudier et suivre la fréquentation dans les espaces littoraux, marins et insulaires? De l'état de l'art à une vision prospective de la recherche}

Nicolas Le Corre, Solenn Le Berre, Louis Brigand et Ingrid Peuziat

\section{(2) OpenEdition \\ Journals}

Édition électronique

URL : https://journals.openedition.org/echogeo/12749

DOI : $10.4000 /$ echogeo. 12749

ISSN : 1963-1197

Éditeur

Pôle de recherche pour l'organisation et la diffusion de l'information géographique (CNRS UMR 8586)

Référence électronique

Nicolas Le Corre, Solenn Le Berre, Louis Brigand et Ingrid Peuziat, « Comment étudier et suivre la

fréquentation dans les espaces littoraux, marins et insulaires?

De l'état de l'art à une vision prospective de la recherche », EchoGéo [En ligne], 19 | 2012, mis en ligne le

10 février 2012, consulté le 10 août 2021. URL : http://journals.openedition.org/echogeo/12749 ; DOI : https://doi.org/10.4000/echogeo.12749

Ce document a été généré automatiquement le 10 août 2021.

EchoGéo est mis à disposition selon les termes de la licence Creative Commons Attribution - Pas d'Utilisation Commerciale - Pas de Modification 4.0 International (CC BY-NC-ND) 


\title{
Comment étudier et suivre la
} fréquentation dans les espaces littoraux, marins et insulaires ? De l'état de l'art à une vision prospective de la recherche

\author{
Nicolas Le Corre, Solenn Le Berre, Louis Brigand et Ingrid Peuziat
}

\section{Introduction}

1 Les littoraux français sont aujourd'hui le siège d'activités anthropiques multiples, parfois concurrentes, interagissant avec l'environnement naturel, dont la conservation est au cœur des préoccupations des gestionnaires. En témoignent le nombre d'aires marines et littorales protégées existantes ou en projet (réserves et parcs marins), mais également la mise en place de Natura 2000 en mer et l'affectation progressive du domaine public maritime au Conservatoire du littoral.

2 L'essor du tourisme et plus spécialement du tourisme de nature, génère un attrait grandissant pour ces espaces protégés. Ce mode de fréquentation, qui se revendique respectueux de l'environnement, génère cependant des conséquences pouvant se traduire par des dégradations comme celles liées au piétinement de la flore, au dérangement de la faune, ou à la pollution des habitats (Le Berre, 2008). Au-delà des préoccupations environnementales, les conflits d'usages apparaissent souvent inévitables (Duhamel et al., 2002).

3 Pourtant, le tourisme représente une activité économique majeure. De son développement dépend le maintien d'une large partie des populations locales. Dans ce contexte, comment concilier tourisme et gestion de l'environnement naturel? Comment assurer l'avenir économique et social de tels territoires? Ces questions sont au cœur d'une problématique de développement durable, déclinée sur les littoraux au 
travers du concept de gestion intégrée de la zone côtière. La demande de nouveaux principes de gestion des espaces sensibles est actuellement forte. En ce sens, la définition de seuils d'acceptabilités d'ordres sociaux, économiques et environnementaux permettrait de fonder et d'appuyer les prises de décisions des gestionnaires (Brigand, Le Berre, 2007 ; Brigand, Le Berre, 2009).

4 La définition de ces seuils pose immanquablement la question des outils et des méthodes à mettre en œuvre pour étudier et suivre les activités touristiques, récréatives mais aussi professionnelles sur les espaces naturels, protégés ou non. Malgré un intérêt grandissant des scientifiques et des gestionnaires pour l'étude de la fréquentation humaine dans les espaces de nature, on constate qu'aucune revue de littérature d'envergure n'a encore été réalisée sur cette thématique, ce qui permettrait pourtant de mettre en évidence les points forts mais aussi les lacunes de la recherche française ainsi que les champs d'investigation scientifiques restant à explorer dans le domaine des sciences humaines et sociales.

\section{Objectifs de l'état de l'art et modalités de la recherche bibliographique}

\section{Objectifs de la recherche bibliographique}

5 Le travail présenté est une synthèse d'une réflexion menée dans le cadre d'une convention de recherche associant deux équipes, l'une en géographie (laboratoire Géomer LETG UMR 6554 CNRS) l'autre en économie (laboratoire AMURE) et l'Agence des Aires Marines Protégées. L'objectif consiste à recenser et analyser, à travers une revue bibliographique, les méthodes d'étude et de suivi ainsi que les observatoires de la fréquentation appliqués au domaine marin (côtier et large) et aux espaces insulaires et littoraux terrestres français. La notion de fréquentation est envisagée au sens large : elle inclut les usages récréatifs, touristiques et professionnels. Les résultats de cet état de l'art, au regard de la durée de l'étude (six mois) et de la thématique qui est très large et ouverte, ne peuvent prétendre à l'exhaustivité. Il s'agit d'une première contribution, dans le domaine des sciences humaines et sociales. Les méthodologies et les observatoires portant sur les retombées socio-économiques de la fréquentation ne sont pas présentés dans cet article. Cependant, l'ensemble des résultats figurent dans un rapport détaillé (Le Corre et al., 2011).

\section{Modalités de la recherche bibliographique}

6 La recherche s'est appuyée sur 20 bases bibliographiques ${ }^{1}$ (nationales et internationales, généralistes et spécialisées) et sur 22 mots-clés se rapportant à notre objet de recherche (figure 1). Un système de croisement se basant sur 192 combinaisons de mots-clés a été retenu et systématiquement utilisé afin de traiter chaque base de données bibliographiques. Le temps de prospection a été quantifié à 88 jours pour 1 personne.

7 D'un point de vue strictement quantitatif, ce sont 258917 résultats bruts qui ont été examinés mais ce sont 1047 références qui ont été jugées pertinentes au regard de notre problématique, ce qui correspond à une moyenne de seulement $2 \%$ de références 
pertinentes (les $98 \%$ restants correspondent, soit à des références jugées à la marge de notre recherche, soit à des références totalement hors sujet).

Figure 1 - Le système de croisement des mots-clés

\begin{tabular}{|c|c|c|}
\hline $\begin{array}{c}\text { Comment } \\
\text { (dispositifs et méthodes } \\
\text { de suivi) }\end{array}$ & $\begin{array}{c}\text { Quoi } \\
\text { (usages et activités } \\
\text { humaines) }\end{array}$ & $\begin{array}{c}\text { Où } \\
\text { (espaces } \\
\text { considérés) }\end{array}$ \\
\hline Observatoire & Fréquentation & Espaces \\
\hline Suivi & Activités humaines & Espaces naturels \\
\hline Etude de fréquentation & Usages & Mer \\
\hline Indicateur & Capacité de charge & Marin \\
\hline Enquête & Pêche à pied & Littoral \\
\hline \multirow{5}{*}{ Système d'information } & Plaisance & Ile \\
\hline & Nautisme & \\
\hline & Plongée & \\
\hline & Loisirs & \\
\hline & Activités récréatives & \\
\hline
\end{tabular}

\section{Revue de littérature portant sur les méthodes d'étude et de suivi de la fréquentation}

8 L'état de l'art vise, dans un premier temps, à porter à connaissance les outils disponibles en matière d'étude de la fréquentation et à envisager leur pertinence, leur opérationnalité et leur possible reproductibilité d'un site à l'autre (Le Corre et al., 2011). Cette analyse se base essentiellement sur les études scientifiques françaises avec des références aux études non scientifiques et étrangères.

\section{Les méthodes identifiées}

9 Les sciences sociales mettent à disposition trois grandes familles d'études qui correspondent chacune à des questionnements bien spécifiques et apportent des réponses différentes mais complémentaires (Le Berre, 2008 ; Peuziat, 2005). Les études quantitatives qui répondent essentiellement aux questions «combien?» «où?», «quand?». Les études qualitatives qui répondent aux questions "qui ? ", «quoi?", "pourquoi?». Les études comportementales qui répondent à la question « comment?».

Les méthodes visant à quantifier la fréquentation des espaces marins, littoraux et insulaires

10 Les données sur le nombre de visiteurs et d'usagers qui fréquentent un site constituent généralement une information fondamentale. L'approche quantitative, qui apporte des chiffres, révèle l'intensité de la fréquentation des espaces étudiés. Elle permet de connaître sa répartition et ses variations dans l'espace et dans le temps, mais aussi de qualifier les usages dans certains cas. Les méthodes de collecte de données quantitatives sont le plus souvent basées sur des comptages. 
Deux types de comptages peuvent être distingués (tableaux 1 et 2) :

- Les comptages directs, qui reflètent la réalité de terrain. Certains de ces comptages livrent des données statiques, qui permettent d'obtenir une vision instantanée de la fréquentation à un instant T. D'autres livrent des données dynamiques, de type «flux de fréquentation ».

- Les comptages indirects, qui révèlent des tendances, des ordres de grandeurs, ou une évaluation relative de la fréquentation. Ils sont généralement basés sur des extrapolations à partir de données détournées de leur utilisation d'origine (données de gestion comptables, indices de présence humaine sur un site, etc.).

Tableau 1 - 16 méthodes de comptages sur les espaces littoraux et insulaires terrestres

Méthodes de comptages directes

\section{- Comptages manuels par du personnel sur site}

Le comptage est réalisé directement sur le site par une équipe de scientifiques ou d'agents à partir de points fixes ou en se déplaçant le long d'un itinéraire prédéterminé. La donnée peut être enregistrée par des compteurs à main ou reportée sur des supports adaptés (fiches d'observation, dictaphones, etc.) avant d'être intégrée à une base de données.

- Survols avec prises de vues photographiques

Lors d'un survol de l'espace étudié et à partir d'un plan de vol prédéfini, un observateur réalise des photographies afin d'identifier et de quantifier les activités humaines. Les clichés sont ensuite photo-interprétés; les données sont généralement intégrées à un SIG ou à une autre base de données adaptée (Excel, Access, etc.).

- Traitement des images satellitaires

La méthode propose de s'appuyer sur l'interprétation des images satellitaires afin de caractériser et quantifier la fréquentation terrestre.

- Comptages manuels des visiteurs lors des débarquements sur les îles ou les îlots

Il s'agit de dénombrer et d'identifier les passagers (excursionnistes, séjournants et insulaires) venus sur les îles à bord des bateaux de transport maritime ou des embarcations de plaisance.

- Comptages automatiques à partir de matériels spécialisés (capteurs infrarouge, dalles acoustiques, etc.)

Le comptage est réalisé à partir d'appareils choisis et disposés de façon stratégique sur l'ensemble du site en fonction des usages que l'on souhaite étudier (trafic routier, trafic de vélos, trafic piétons). Les données sont recueillies en continu tout au long de la durée de fonctionnement des appareils.

- Comptages à partir de photographies automatiques ou de caméras vidéo

L'appareil photographique ou la caméra vidéo sont disposés de façon stratégique sur le site. Ils fonctionnent en continu ou se déclenchent lors du passage des visiteurs.

Méthodes de comptages indirectes 


\section{- Evaluation de la fréquentation par indices de présence}

Méthode d'évaluation de la pression de la fréquentation qui s'appuie sur les indices et les signes laissés par les visiteurs sur le site : traces de passage, piétinement de la flore, érosion des sentiers, dépôt de déchets, etc. L'évaluation est réalisée sur le site à partir d'observations directes ou à partir de photographies aériennes.

- Traitement des données fiscales relatives à la taxe sur les passagers maritimes (« taxe Barnier »)

En s'appuyant sur les recettes fiscales perçues par les douanes par le biais de la taxe Barnier, il est possible d'extrapoler le nombre de visiteurs à avoir emprunté des navettes pour accéder à certains espaces naturels protégés.

- Traitement des données de gestion comptables produites par les gestionnaires

Il s'agit de s'appuyer sur les données générales produites naturellement par certaines structures-gestionnaires: vente de tickets d'entrées, nombre de sorties organisées, réservations, etc.

- Traitement des données de gestion comptables produites par les transporteurs maritimes

Méthode basée sur les données officielles produites par les transporteurs maritimes (notamment le nombre d'achat de billets d'embarquement) pour évaluer les variations de la fréquentation sur les îles.

- Traitement des données relatives aux permis et aux licences de pratique d'une activité professionnelle, récréative ou touristique

La pratique de certaines activités professionnelles, récréatives ou touristiques nécessite parfois de disposer d'un permis ou d'une licence (ex : chasse). Dans certains cas, ce sont les aires protégées elles-mêmes qui imposent ces autorisations. Le traitement de ces données permet alors de disposer d'informations générales sur l'évolution temporelle et quantitative d'une activité.

- Traitement des données des téléphones portables

Sur le principe, les téléphones portables s'apparent à des transpondeurs. Chaque appareil détient un code unique (ID) et renvoie en continu un signal aux antennes relais les plus proches. En s'appuyant sur ces informations, il est alors possible de quantifier mais également de géolocaliser, les propriétaires de téléphones portables dans un espace considéré.

- Enquêtes par voie postale, électronique ou par internet

Enquêtes réalisées à petite échelle qui permet d'estimer la proportion de la population d'un pays, d'une région ou d'un département qui fréquentent un territoire ou un milieu spécifique (ex: la forêt, le bassin d'Arcachon) ou qui pratiquent une activité de loisir particulière (ex : la pêche à pied, la plaisance).

- Quantifier la fréquentation par la technique de l'intelligence artificielle

Développée par le bureau d'étude Usages et Territoires, cette approche s'appuie sur un logiciel de data mining utilisant des techniques d'intelligence artificielle. Elle a permis de valoriser et de modéliser des données empiriques (issues d'enquêtes qualitatives auprès des gardes du littoral) pour évaluer la fréquentation sur l'ensemble des sites du Conservatoire du littoral. 
- Méthode de la variation de consommation de farine

La méthode part du constat que le pain est«d'un usage indispensable, fréquent et constant ". Aussi, elle propose de s'appuyer sur les consommations de farine afin de mettre en évidence les variations de la fréquentation touristique sur un territoire donnée (une région, un département).

- Inscription volontaire sur les registres des aires protégées

Traitement des données fournies par les visiteurs eux-mêmes sur les livres d'or, les livres de suggestions disponibles le long du parcours dans les espaces protégés (accueils, gîtes, points d'information, etc.)

Source : recensement opéré à partir des bases de données bibliographiques suivantes : Academic Search Premier, Archimer, Asfa, Avano, Cairn, Catalogue La Pérouse, DOAJ, Google, Google Scholar HAL Jstor, Pascal, Persée, Revue.Org, Science Direct, Scirus, SpringerLink, Thèse En Ligne, Web of Science, Wiley-Blackwell.

Tableau 2 - 14 méthodes de comptages sur les espaces strictement marins

\section{Méthodes de comptages directes}

- Comptages manuels des activités nautiques ou des bateaux au mouillage

Des comptages manuels sont réalisés pour identifier et quantifier les activités nautiques ou le nombre d'embarcations au mouillage près des littoraux, autour des îles ou dans les archipels. Selon les caractéristiques des sites, les observations sont effectuées depuis la terre ferme ou à bord d'un moyen à la mer.

- Comptages manuels des flux d'embarcations sur les cales de mise à l'eau

Méthode qui consiste à identifier et à quantifier les bateaux sur remorque mis à l'eau à partir des cales situées sur le continent, et à évaluer le trafic maritime lié aux bateaux sur remorque.

- Comptages manuels des flux maritimes à la sortie des ports

Méthode qui consiste à identifier et à quantifier les embarcations actives à leur sortie du port, et le trafic maritime qu'elles génèrent.

- Survols avec prises de vue photographiques des activités nautiques ou des mouillages littoraux et insulaires

Lors d'un survol prédéfini de l'espace littoral (plages, espace marin proche du contient, etc.), un observateur réalise des photographies de l'espace marin ou du linéaire côtier afin d'identifier et de quantifier les activités nautiques ou les mouillages.

- Survols avec localisation des navires en mer par GPS

Lors d'un survol prédéfini de l'espace maritime, l'identification et la localisation de tous les navires se font en direct grâce à un GPS. Les données sont ensuite reportées dans un SIG. 
- Survols avec caméra embarquée

Lors d'un survol prédéfini de l'espace maritime, une caméra très haute définition permet de filmer et de géo-référencer en direct l'ensemble des navires présents sur un bassin maritime. La caméra est directement téléguidée par un assistant qui peut, au besoin, zoomer sur des zones spécifiques.

- Traitement des images satellitaires

La méthode propose de s'appuyer sur l'interprétation des images satellitaires afin de caractériser et quantifier la fréquentation nautique.

- Comptages automatiques à partir de matériels spécialisés (capteurs infrarouge, etc.)

Ces capteurs ont été développés, à l'origine, pour compter les visiteurs sur les milieux terrestres. Certains auteurs et sociétés privées envisagent d'adapter ces procédés aux espaces marins, notamment pour évaluer les flux maritimes à l'entrée des ports ou des baies, dans certains chenaux, entre des îles.

- Traitement des données Radar et Sonar

Méthodes qui proposent de s'appuyer sur les données Sonar ou Radar afin de caractériser et quantifier la fréquentation nautique.

\section{Méthodes de comptages indirectes}

- Traitement des données de gestion comptables produites par les transporteurs maritimes

Méthode basée sur les données officielles produites par les transporteurs maritimes (notamment le nombre d'achat de billets d'embarquement et le nombre de rotations des navires) pour évaluer l'intensité du trafic maritime organisé et les circuits empruntés.

- Traitement des données relatives aux permis et aux licences de pratique d'une activité professionnelle, récréatives ou touristique

La pratique de certaines activités professionnelles, récréatives ou touristiques nécessite parfois de disposer d'un permis ou d'une licence (ex : plaisance à moteur, scooter des mers, chasse sous-marine, charte de plongée). Dans certains cas, ce sont les aires protégées elles-mêmes qui imposent et délivrent ces autorisations. Le traitement de ces données permet alors de disposer d'informations générales sur l'évolution quantitative et temporelle du nombre de pratiquants.

- Traitement des données des sémaphores

Dans le cadre de leurs activités de surveillance maritime, les sémaphoristes relèvent en continu des informations sur les navires en mer. La méthode consiste à s'appuyer sur ces informations de façon à caractériser et à quantifier les flux de navires sur le bassin maritime étudié.

\section{- Traitement des données AIS (Automatic Identification System)}

L'AIS est un système d'information opérationnel, en temps réel, destiné à la sécurité et la surveillance du trafic maritime, à l'échelle nationale mais également internationale. Il est imposé à certains navires par la convention Solas. En s'appuyant sur ces données (mais en les détournant de leur utilisation d'origine), il est possible de caractériser, de quantifier et de localiser les flux maritimes mais également de décrire l'emprise spatio-temporelle des navires à l'échelle d'un site d'étude. 


\section{- Enquêtes par voie postale, électronique ou par internet}

Enquêtes réalisées à petite échelle qui permet d'estimer la proportion de la population d'un pays, d'une région ou d'un département qui fréquente un territoire ou un milieu naturel spécifique (ex : un littoral) ou qui pratiquent une activité de loisir particulière.

Source : recensement opéré à partir des bases de données bibliographiques suivantes: Academic Search Premier, Archimer, Asfa, Avano, Cairn, Catalogue La Pérouse, DOAJ, Google, Google Scholar, HAL Jstor, Pascal, Persée, Revue.Org, Science Direct, Scirus, SpringerLink, Thèse En Ligne, Web of Science, Wiley-Blackwell.

\section{Les méthodes visant à qualifier la fréquentation des espaces marins, littoraux et insulaires}

12 Si les données chiffrées sont indispensables, elles n'ont que peu de sens si elles ne sont pas mises en perspective avec des données qualitatives. Seules ces dernières permettent de caractériser la fréquentation et les profils des usagers, de comprendre les mécanismes de l'opinion, de connaitre les motivations à fréquenter un site, à pratiquer une activité, de mettre en évidence la perception qu'ont les usagers de leur environnement, leurs attentes et leurs propositions, etc. Les méthodes développées, basées sur la réalisation d'enquêtes, relèvent essentiellement de la sociologie, plus rarement de l'anthropologie.

On distingue deux grandes catégories de méthodologies d'enquête, selon l'échelle d'étude choisie (tableau 3):

- les enquêtes à petite échelle, utilisées pour appréhender une question de fréquentation auprès de la population d'un pays, d'une région ou d'un département ;

- les enquêtes à grande échelle, utilisées pour comprendre localement une question de fréquentation auprès de la population d'usagers d'une aire protégée ou d'un territoire de pratique.

Tableau 3 - 4 méthodes d'enquête pour connaître les usagers, les perceptions et les pratiques

\section{Les enquêtes à petite échelle}

- Les enquêtes touristiques (" au domicile », « aux frontières ", « à poids partagé », etc.)

- Les enquêtes par voie postale, électronique ou par internet

Les enquêtes à grande échelle

- Les entretiens (non directif, semi directifs, directifs)

- Les questionnaires standardisés (à questions fermées et/ou à questions ouvertes)

Source : recensement opéré à partir des bases de données bibliographiques suivantes : Academic Search Premier, Archimer, Asfa, Avano, Cairn, Catalogue La Pérouse, DOAJ, Google, Google Scholar, HAL Jstor, Pascal, Persée, Revue.Org, Science Direct, Scirus, SpringerLink, Thèse En Ligne, Web of Science, Wiley-Blackwell. 


\section{Les méthodes visant à caractériser les comportements des usagers sur les sites}

L'approche comportementale des usages est relativement rare. Elle est essentiellement basée sur des observations participantes ou non, impliquant le plus souvent une forte immersion du chercheur sur le site d'étude (tableau 4). Elle prend en compte de façon approfondie la diversité des acteurs et de leurs pratiques, apportant les clés de compréhension des phénomènes de fréquentation indispensables à la caractérisation fine des systèmes de fréquentation. Ces observations permettent notamment de saisir comment les usagers agissent (et interagissent entre eux) sur un site donné, comment ils se comportent dans leur environnement, comment ils utilisent et s'approprient l'espace. La mise en œuvre de l'approche comportementale apporte des informations et un cadre d'échanges particulièrement intéressants dans les réflexions sur la gouvernance des sites protégés.

Tableau 4 - 10 méthodes pour comprendre le comportement des usagers

\section{Les analyses directes}

- Les observations comportementales des différentes familles d'usagers

Les observations comportementales permettent de comprendre comment les visiteurs agissent (et interagissent entre eux), comment ils se comportent dans leur environnement, comment ils utilisent l'espace. Elles visent à définir des lois de comportements dans un lieu donné, lois qui peuvent être éventuellement étendues et généralisées.

- Les instantanés cartographiques

Les instantanés cartographiques (fonds de carte sur lesquels sont reportées des données de fréquentation à un instant $\mathrm{T}$ ) sont généralement utilisés pour cartographier puis quantifier les activités humaines présentes sur un site. Ces derniers peuvent également servir de base à une étude sur le comportement des visiteurs si les informations qui y sont annotées sont suffisamment précises (activités pratiquées, attitudes, direction, etc.).

\section{- Les Suivis GPS}

Méthode qui consiste à demander à une ou plusieurs personnes de bien vouloir enregistrer leur itinéraire pendant leur temps de présence sur un site grâce à un GPS de poche.

- Les reconstitutions d'itinéraires (à partir d'enquêtes)

S'appuyant sur des enquêtes de terrain (entretiens semi-directifs, QCM), les observateurs demandent aux visiteurs de reconstituer eux-mêmes (sur un fond de carte prévu à cet effet), l'itinéraire qu'ils ont emprunté pendant leur visite.

\section{- L'utilisation de la vidéo}

Si la vidéo est d'abord citée pour quantifier la fréquentation d'un site (sentiers, cales, plages), certains auteurs proposent de s'appuyer sur cette méthode pour mener des études comportementales.

Les analyses indirectes 


\section{- Les carnets d'observations}

Il s'agit de proposer à certains visiteurs et usagers de sites naturels de se munir d'un carnet d'observations (ex : carnet de pêche). Ces derniers s'engagent alors à y annoter, par eux-mêmes, des informations relatives à leurs sorties sur le site étudié : itinéraire emprunté, activités pratiquées, groupes d'usagers rencontrés, etc. Dans la plupart des cas, les enquêtés conservent leur carnet pendant une période définie à l'avance (1 semaine, 1 mois, etc.).

- Traitement des données AIS (Automatic Identification System)

L'AIS est un système d'information opérationnel, en temps réel, destiné à la sécurité et la surveillance du trafic maritime, à l'échelle nationale mais également internationale. Il est imposé à certains navires par la convention Solas. En s'appuyant sur ces données et en les détournant de leur utilisation d'origine, il est possible de caractériser, de quantifier et de localiser des voies de navigation sur un site donnée.

\section{- Utilisation des « boîtes noires »}

Testé en Australie, le système des «boîtes noires » s'apparente au matériel embarqué sur les avions. Ce dernier enregistre un certain nombre de paramètres (mouvements, trajectoires, vitesse) sur les bateaux équipés. Il permet également d'y enregistrer, grâce à un petit ordinateur de bord, des données relatives aux interactions avec la faune marine observée (espèces, nombre d'individus, localisation, date et heure de l'observation, etc.)

\section{- Utilisation de l'I-Tracker}

Testé aux Etats-Unis, le I-Tracker est un ordinateur portable ultra-léger qui utilise un logiciel gratuit (Cyber Tracker) adapté à l'acquisition de données environnementales. Le système est aujourd'hui détourné de son utilisation initiale et adapté pour suivre le comportement des visiteurs ou des professionnels du tourisme (mouvements, trajectoires, vitesse des personnes ou des navires). Les visiteurs équipés y enregistrent également des informations relatives à la faune marine qu'ils observent (espèces, nombre d'individus, lieu et heures, etc.).

\section{- L'utilisation des données des téléphones portables}

Sur le principe, les téléphones portables s'apparent à des transpondeurs. Chaque appareil détient un code unique (ID) et renvoie en continu un signal aux antennes relais les plus proches. En s'appuyant sur ces informations, il est alors possible de quantifier mais également de géolocaliser les propriétaires de téléphones portables dans un espace considéré.

Source : recensement opéré à partir des bases de données bibliographiques suivantes : Academic Search Premier, Archimer, Asfa, Avano, Cairn, Catalogue La Pérouse, DOAJ, Google, Google Scholar, HAL Jstor, Pascal, Persée, Revue.Org, Science Direct, Scirus, SpringerLink, Thèse En Ligne, Web of Science, Wiley-Blackwell.

\section{Analyse critique des méthodes d'étude et de suivi de la fréquentation}

\section{Intérêts et points forts}

La revue de littérature met en évidence un foisonnement des méthodes ( 44 familles au total) pour étudier et suivre les usages récréatifs et touristiques dans les espaces de 
nature. Ce constat est d'autant plus intéressant que ces dernières sont variées et complémentaires, laissant envisager pour le scientifique, comme pour le gestionnaire, de multiples possibilités d'adaptation face à des sites aux caractéristiques géographiques, aux enjeux et aux questionnements de fréquentation souvent singuliers. De nombreuses études ont également démontré leur grand intérêt (scientifique et opérationnel), soit par leur aptitude à tester des méthodes innovantes et produire des résultats inédits, soit par leur capacité à combiner plusieurs méthodes complémentaires pour cerner et décrypter de façon détaillée l'ensemble d'un système de fréquentation donné.

Ce constat sur la littérature française est d'autant plus encourageant que l'engouement des sciences humaines et sociales pour cette thématique de la fréquentation humaine est relativement récent avec un premier essor dans les années 1990 (figure 2). Avant cette date, les études spécialisées étaient ponctuelles (seulement une quinzaine d'études identifiées entre les années 1960 et 1990) et émanaient le plus souvent de structures gestionnaires, les premières à prendre véritablement conscience de la montée de nouveaux enjeux environnementaux et sociaux liés à l'accueil des publics dans les aires protégées. Ainsi, des organismes comme les parcs nationaux ou l'Office National des Forêts ont été des précurseurs contribuant à mettre en œuvre les premières approches quantitatives (comptages) et qualitatives (enquêtes) de la fréquentation dès les années 1960-1970, et ceci bien avant que les scientifiques ne s'y intéressent véritablement. A partir des années 2000 et notamment des années 2005, un deuxième essor se dessine nettement. Avec 24 publications en 2010, cette tendance semble d'ailleurs s'accentuer encore davantage. Cet intérêt peut s'expliquer par plusieurs raisons : des laboratoires qui développent des axes de recherche sur cette thématique; des colloques sur la gestion du littoral qui se sont multipliés ces dernières années et au cours desquels des présentations sur la gestion de la fréquentation ont été présentées ; un intérêt grandissant auprès des étudiants et surtout de la part des gestionnaires qui ont besoin de justifier leurs choix de gestion à partir de données notamment quantitatives. 
Figure 2 - Évolution du nombre de publications scientifiques relatives à l'étude de la fréquentation humaine en France depuis les années 1950

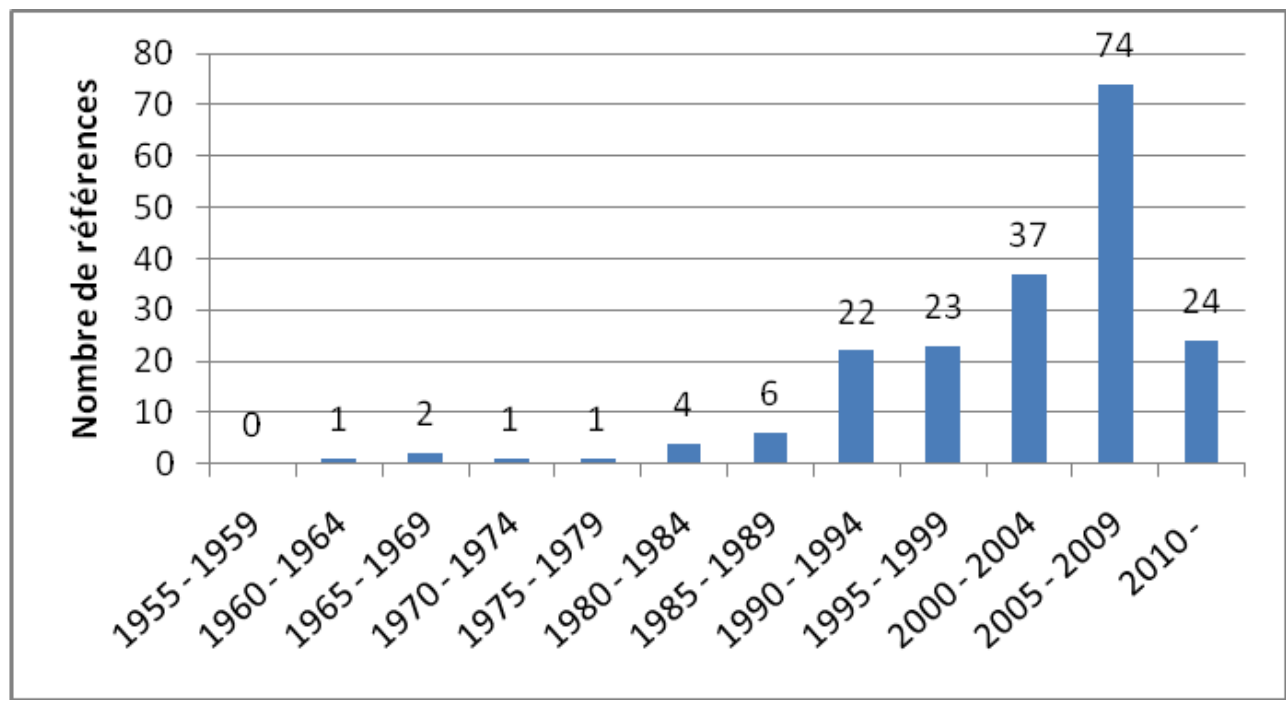

Ne sont pas pris en compte dans le graphique toutes les publications émanant d'organismes non scientifiques. Les données présentées dans cette figure nécessitent d'être interprétées avec prudence. D'une part, il est très probable que tous les domaines scientifiques en lien avec l'environnement aient connu une progression assez similaire en termes de production scientifique. D'autre part, l'accélération de la production scientifique dans les années 2000 doit également être interprétée au regard de la démocratisation des bases de données scientifiques qui permettent une recherche rapide et plus exhaustive (ce qui n'était pas le cas avant les années 2000).

Source : recensement opéré à partir des bases de données bibliographiques suivantes: Academic Search Premier, Archimer, Asfa, Avano, Cairn, Catalogue La Pérouse, DOAJ, Google, Google Scholar, HAL Jstor, Pascal, Persée, Revue.Org, Science Direct, Scirus, SpringerLink, Thèse En Ligne, Web of Science, Wiley-Blackwell.

\section{Analyse de la validité scientifique des méthodes}

Ces aspects positifs ne doivent cependant pas cacher certaines limites et difficultés auxquelles doit faire face la recherche française actuelle.

Tout d'abord, si les méthodes identifiées sont effectivement nombreuses, beaucoup restent encore à développer, à améliorer et à valider. Pour s'en rendre compte, les méthodes recensées ont été classées selon trois degrés de validité scientifique (tableau 5). Ainsi, on distingue :

- 11 méthodes ( $25 \%$ ) dont la pertinence et la validité scientifique sont avérées. Ces méthodes font l'objet de nombreuses études et références bibliographiques, notamment sur les milieux terrestres.

- 18 méthodes (43,9\%) ont été validées, mais par un nombre restreint d'équipes scientifiques. Elles demandent à être affinées et généralisées, notamment en les testant sur un nombre plus important de sites. On peut citer les méthodes de comptage à partir des cales de mises à l'eau, les survols avec utilisation des GPS pour quantifier les embarcations sur un espace maritime, la prise de photographies pour estimer le nombre de personnes sur les plages, le traitement des données des sémaphores pour caractériser les flux maritimes, etc.

- 15 méthodes $(31,1 \%)$ restent encore largement à imaginer, à développer et à valider. Ces méthodes sont de l'ordre de l'expérimental, voire du secteur relevant de la recherche et du développement. Celles-ci sont loin d'être opérationnelles et font l'objet d'un nombre limité d'études. Certaines d'entre elles sont citées comme de simples pistes de réflexions, sans réelle étude appliquée. C'est le cas de la méthode de comptage automatique sur la mer à 
partir de matériels spécialisés (tels que les compteurs « marins »), l'utilisation des radars et des sonars pour quantifier et caractériser les flux des embarcations de plaisance, ou encore le traitement des images satellitaires.

Tableaux $5 a, 5 b, 5 c, 5 d$ - Validité scientifique des méthodes d'étude et de suivi des usages récréatifs et touristiques dans les aires protégées

Critères de validité :

- en vert : méthodes largement testées et validées par la communauté scientifique faisant

l'objet de nombreuses études et références bibliographiques (11, $25 \%)$

- en jaune : méthodes testées et validées par un nombre limité d'équipes scientifiques. Il convient d'être vigilant quant à leur utilisation car elles méritent d'être affinées et testées sur d'autres sites $(18,43,9 \%)$

- en rouge : méthodes non encore testées ou validées scientifiquement. Celles-ci sont encore expérimentales et non opérationnelles. Certaines de ces méthodes n'ont tout simplement jamais fait l'objet d'études de terrain en France (voire à l'étranger) (15, 31,1 \%).

Source : recensement opéré à partir des bases de données bibliographiques suivantes: Academic Search Premier, Archimer, Asfa, Avano, Cairn, Catalogue La Pérouse, DOAJ, Google, Google Scholar, HAL Jstor, Pascal, Persée, Revue.Org, Science Direct, Scirus, SpringerLink, Thèse En Ligne, Web of Science, Wiley-Blackwell.

Tableau 5a - Méthodes de comptages sur les milieux littoraux et insulaires terrestres

\begin{tabular}{|l|l|}
\hline 16 méthodes de comptages sur les milieux littoraux et insulaires terrestres & $\begin{array}{c}\text { Validité } \\
\text { scientifique }\end{array}$ \\
\hline Comptages manuels par du personnel sur site & \\
\hline Traitement des données des transporteurs maritimes & Comptages manuels des visiteurs lors des débarquements sur les îles ou les îlots \\
\hline Comptages automatiques à partir de matériels spécialisés & Traitement des données officielles produites par les gestionnaires \\
\hline $\begin{array}{l}\text { Traitement des données relatives aux permis et aux licences de pratique d'une activité professionnelle, } \\
\text { récretive ou touristique }\end{array}$ & Survols avec prises de vues photographiques \\
\hline Comptages à partir de photographies automatiques ou de caméras-vidéo & \\
\hline Traitement des données fiscales relatives à la taxe sur les passagers maritimes (« taxe Barnier ») \\
\hline Enquêtes par voie postale, électronique ou par internet \\
\hline Traitement des images satellitaires \\
\hline Utilisation des données des téléphones portables \\
\hline Evaluation de la fréquentation par indices de présence \\
\hline Quantification de la fréquentation par la technique de l'intelligence artificielle \\
\hline Méthode de la variation de consommation de farine \\
\hline Inscription volontaire sur les registres des aires protégées \\
\hline
\end{tabular}


Tableau 5b - Méthodes de comptages sur les milieux marins

\begin{tabular}{|l|c|}
\hline 14 méthodes de comptages sur les milieux marins & $\begin{array}{c}\text { Validité } \\
\text { scientifique }\end{array}$ \\
\hline Comptages manuels des activités nautiques ou des bateaux au mouillage & \\
\hline Survols avec prises de vue photographiques des activités nautiques ou des mouillages littoraux et insulaires & \\
\hline Survols avec localisation des navires en mer par GPS & \\
\hline Comptages manuels sur les cales de mise à l'eau & \\
\hline Comptages manuels des flux maritimes à la sortie des ports & \\
\hline Traitement des données des sémaphores & \\
\hline Traitement des données AIS (Automatic Identification System) & \\
\hline $\begin{array}{l}\text { Traitement des données relatives aux permis et aux licences de pratique d'une activité professionnelle, } \\
\text { récréatives ou touristique }\end{array}$ & \\
\hline Enquêtes par voie postale, électronique ou par internet & \\
\hline Comptages automatiques à partir de matériels spécialisés \\
\hline Traitement des données Radar et Sonar \\
\hline Survols avec caméra embarquée \\
\hline Traitement des images satellitaires \\
\hline
\end{tabular}

Tableau $5 c$ - Méthodes d'enquêtes pour connaître les usagers, les perceptions et les pratiques

\begin{tabular}{|l|c|}
\hline 4 méthodes d'enquête pour connaître les usagers, les perceptions et les pratiques & $\begin{array}{c}\text { Validité } \\
\text { scientifique }\end{array}$ \\
\hline Les enquêtes touristiques ( « au domicile », « aux frontières », « à poids partagé », etc.) \\
\hline Les entretiens (non directif, semi directifs, directifs) \\
\hline Les questionnaires standardisés (à questions fermées et/ou à questions ouvertes) \\
\hline Les enquêtes par voie postale, électronique ou par internet \\
\hline
\end{tabular}

Tableau 5d - Méthodes pour comprendre le comportement des usagers

\begin{tabular}{|l|c|}
\hline $\mathbf{1 0}$ méthodes pour comprendre le comportement des usagers & $\begin{array}{c}\text { Validité } \\
\text { scientifique }\end{array}$ \\
\hline Les observations de type ethnographique des différentes familles d'usagers & \\
\hline Les carnets d'observations & \\
\hline Les instantanés cartographiques & \\
\hline Les Suivis GPS & Les reconstitutions d'itinéraires (à partir d'enquêtes) \\
\hline L'utilisation de la vidéo \\
\hline Traitement des données AIS (Automatic Identification System) \\
\hline Utilisation des « boites noires » \\
\hline Utilisation de l'I-Tracker \\
\hline L'utilisation des données des téléphones portables \\
\hline
\end{tabular}

20 D’un point de vue général, le classement réalisé permet de distinguer les méthodes «traditionnelles» qui constituent généralement les fondements des études de fréquentation (comptages manuels et automatiques in situ, questionnaires, entretiens) des méthodes plus originales mais qui sont par nature moins développées et qui demandent un renforcement de leur cadre méthodologique.

21 D'un point de vue géographique, ce constat se traduit par un certain contraste entre les espaces terrestres et marins. Sur les espaces littoraux et insulaires terrestres, les méthodes sont davantage validées et abouties. En effet, bien que ces derniers présentent un certain nombre de spécificités, les comptages de fréquentation qui peuvent y être appliqués, bénéficient d'une longue expérience menée sur les sites naturels terrestres. 
l'effort de recherche à l'échelle française. Certains sites locaux sont fortement étudiés et des moyens conséquents y sont déployés (à titre d'exemples : Audouit, 2009 ; BaronYellès, 1997 ; Brigand L. et Le Berre, 2009 ; Chlous-Ducharme, F., et al., 2008 ; David et al., 2008 ; Duvat et Mossot, 2011 ; Evrard, 2010 ; Fleury et cadet, 2010 ; Jollit, 2010 ; Le Berre, 2008; Musard, 2003; Parrain, 2010 ; Peuziat, 2005; Picon, 1987; Richez, 1995; Thomassin, 2011; Trouillet, 2006; Vacher et al., 2011). A l'inverse, il reste de nombreuses régions littorales délaissées malgré des enjeux de recherche et de gestion, a priori, tout aussi importants. Les régions caractérisées par un grand nombre d'études (à savoir la Manche, la région Bretagne et plus particulièrement le Finistère, la Charente-Maritime dont l'île d'oléron, les Bouches-du-Rhône, le Var, et dans une moindre mesure la Réunion et la Nouvelle-Calédonie) sont à mettre en lien avec la proximité géographique d'universités et d'organismes de recherche qui se sont spécialisés sur cette thématique: le laboratoire Géomer à l'Université de Bretagne Occidentale (Brest), IFREMER (Brest, la Réunion), le laboratoire LIENSs à l'Université de la Rochelle, le laboratoire ART-Dev à l'Université Paul Valéry (Montpellier), l'IRD à Montpellier, à la Réunion, en Nouvelle-Calédonie, etc. Ces équipes de recherche spécialisées sont peu nombreuses ${ }^{2}$, ce qui explique la distribution très partielle des études sur les façades littorales françaises.

\section{L'absence de réelle pluridisciplinarité}

Si la géographie représente, de loin, la discipline la plus représentée dans l'étude et le suivi de la fréquentation humaine dans les espaces littoraux, marins et insulaires, elle n'en a cependant pas l'hégémonie. Ainsi, des disciplines comme la sociologie, l'ethnographie, l'anthropologie, l'économie mais également la biologie et l'écologie (à travers des organismes comme l'IFREMER ou l'IRD) traitent également cette thématique. Il n'en reste pas moins que l'ensemble des études recensées est caractérisé par l'absence de pluridisciplinarité, y compris au sein des Sciences Humaines et Sociales, avec un effort de recherche global qui reste dispersé mais aussi cloisonné en fonction des sites et des thématiques abordées. En effet, si les collaborations sont nombreuses entre les laboratoires de recherche et les structures gestionnaires, en revanche, il n'en est pas de même entre laboratoires de disciplines pourtant voisines. Peu d'études font ainsi appel à des spécialistes de disciplines différentes avec pour objectifs la transversalité et la mutualisation des compétences. 


\section{L'absence d'un caractère longitudinal} recherche spécialisée est réelle. En effet, exceptions mises à part, les chercheurs privilégient les études de cas sur des sites ateliers à des instants $\mathrm{T}$, plutôt que la recherche de protocoles harmonisés et construits dans la durée, ce qui permettrait de réaliser des analyses inter-sites ou de mettre en lumière des tendances à l'échelle de l'ensemble des aires protégées. Cette absence d'une réflexion globale, à petite échelle, explique en partie, la faiblesse du nombre de références méthodologiques et plus particulièrement de guides généraux, notamment à destination des gestionnaires pour étudier et suivre la fréquentation humaine. Si les études de cas issus de travaux émanant de scientifiques sont nombreuses, en revanche, l'expérience et les compétences capitalisées par les uns et les autres, ne font l'objet d'aucun document de référence (en termes de méthodes, de protocoles, etc.), encore moins d'une véritable stratégie de transfert de connaissances concrètes vers les structures gestionnaires.

\section{L'absence de valorisation}

Enfin, en ce qui concerne la production scientifique proprement dite, on est frappé par l'absence de valorisation des études de terrain dans des revues scientifiques internationales, mais également par la faible valorisation dans des revues françaises référencées par l'AERES. En effet, la production scientifique actuelle prend essentiellement la forme de mémoires de master, de thèses, de rapports, d'actes de colloque, de brèves publications dans les «travaux scientifiques " de certaines aires protégées, de restitutions de programmes scientifiques, mais beaucoup plus rarement d'articles dans des revues à comité de lecture. Ce constat s'explique-t-il par l'absence de reconnaissance au sein des lignes éditorialistes des différentes revues françaises ou encore par la difficulté des chercheurs français en sciences humaines à se conformer aux exigences des revues anglo-saxonnes? Ne doit-on pas tout simplement évoquer un engouement et un intérêt encore trop récents de la part des scientifiques pour la thématique des usages récréatifs et touristiques? Ou par le fait que les chercheurs ne trouvent pas le temps de la valorisation au terme d'une étude de fréquentation, qui a exigé plusieurs mois de terrain et de rédaction?

\section{Une situation qui évolue partiellement}

La situation semble cependant quelque peu évoluer si l'on tient compte de certaines initiatives locales mais aussi régionales et nationales qui traduisent un intérêt pour la thématique. Ainsi, par exemple, le programme PAMPA, initié par IFREMER dans le cadre du programme national de recherche Liteau, a permis de tester des métriques de suivi de la fréquentation dans plusieurs aires marines protégées (en France métropolitaine mais également à la Réunion ou en Nouvelle-Calédonie). De même, la récente formation dispensée par le laboratoire Géomer sur le suivi de la fréquentation touristique auprès des gestionnaires des aires marines protégées de la façade nord de la Méditerranée (réseau MedPAN Nord) (formation qui a eu lieu en juin 2011) donnera lieu en 2012 à la publication d'un guide méthodologique. Citons enfin, la multiplication des études de cas dans les pertuis charentais (laboratoire LIENs), ou encore le projet LIFE LAG'Nature qui a pour objectif une standardisation des protocoles de collecte de 
données de fréquentation pour l'ensemble du réseau d'espaces naturels du littoral de Languedoc-Roussillon.

\title{
Recensement et analyse des dispositifs de suivi de la fréquentation existants en France
}

\author{
Définition et cadre de la mise en place des observatoires de la \\ fréquentation
}

La revue de littérature met en évidence que les méthodes d'étude des usages récréatifs et touristiques ne sont généralement employées que dans le cadre d'études ponctuelles. Pourtant seuls des suivis menés sur le long terme, à partir de la définition d'un état de référence, permettent de comprendre et de qualifier de façon précise et objective des tendances et des changements dans les caractéristiques de la fréquentation des sites mais également de juger de l'efficacité des mesures de gestion mises en œuvre sur le terrain (Wardell, Moore, 2004). La mise en place d'un observatoire de la fréquentation peut constituer un aboutissement logique et parfois nécessaire (Le Berre, 2008).

Les observatoires sont des structures de centralisation et de gestion d'indicateurs essentiellement humains et sociaux (parfois associés à des indicateurs environnementaux) au service de la connaissance et de la protection de sites naturels, historiques ou culturels, et des populations qui les fréquentent et en vivent. Ils visent à étudier et analyser la fréquentation humaine dans ses aspects quantitatifs (pour connaître et suivre l'évolution spatiale et temporelle du nombre de visiteurs et d'usagers sur un site considéré), dans ses aspects qualitatifs (pour connaître et suivre l'évolution des publics notamment leurs profils, leurs attentes, leurs perceptions, etc.), dans ses aspects comportementaux (pour connaître et suivre les attitudes et actions des familles d'usagers sur les sites) et dans ses aspects économiques (notamment pour connaître et suivre les retombées économiques de la fréquentation). Les observatoires peuvent être des outils de recherche et/ou avoir une vocation opérationnelle (gestion de la fréquentation, limitation des impacts environnementaux et/ou sociaux, aide aux politiques publiques).

31 Le travail de recensement des dispositifs de suivi de la fréquentation (à partir de la même méthodologie, cf. paragraphe sur les modalités de la recherche bibliographique) a permis d'identifier 18 observatoires opérationnels sur les espaces littoraux, marins ou insulaires français. Les dispositifs recensés ont été classés en fonction de l'échelle de suivi (locale, régionale ou nationale) et du nombre d'activités et d'usages étudiés (permettant de distinguer les observatoires mono et multi-usages) (carte 1).

\section{Présentation générale des observatoires de la fréquentation en France}

\section{Répartition géographique}

D'un point de vue géographique (carte 1), les dispositifs de suivi de la fréquentation sont présents sur les trois façades maritimes métropolitaines : 3 observatoires sur la façade manche, 5 sur la façade atlantique et 7 sur la façade méditerranéenne. En 
revanche, l'Outre-Mer est très peu représentée avec seulement un observatoire identifié en Guadeloupe (dans le Parc national de Guadeloupe).

Ces observatoires de la fréquentation émanent le plus souvent de la volonté d'une structure gestionnaire en charge d'une aire naturelle sensible, le plus souvent protégée :

- Natura 2000 : Bountîles Chausey et Bountîles Saint-Riom

- Parcs nationaux : Bountîles Pors-Cros et Bountîles Porquerolles, Observatoire de la biodiversité et des usages marins et littoraux, Parc national de la Guadeloupe

- Conservatoire du littoral : archipel de Riou et Bountîles Chausey

- Réserve naturelle nationale : archipel de Riou

- sites de ONF : «Plans Plages » Gironde

- et autres aires protégées : Parc Marin de la côte Bleue, archipel du Frioul.

Les autres dispositifs concernent un monument culturel (Bountîles Mont-Saint-Michel), une île privée (Bountîles Saint-Riom) et deux dispositifs de la fréquentation qui concernent des espaces littoraux non protégés (notamment des estrans) de l'île d'Oléron (GIPREOL et Qualiplages Oléron).

Carte 1 - Les observatoires opérationnels de la fréquentation dans les espaces littoraux, marins et insulaires français

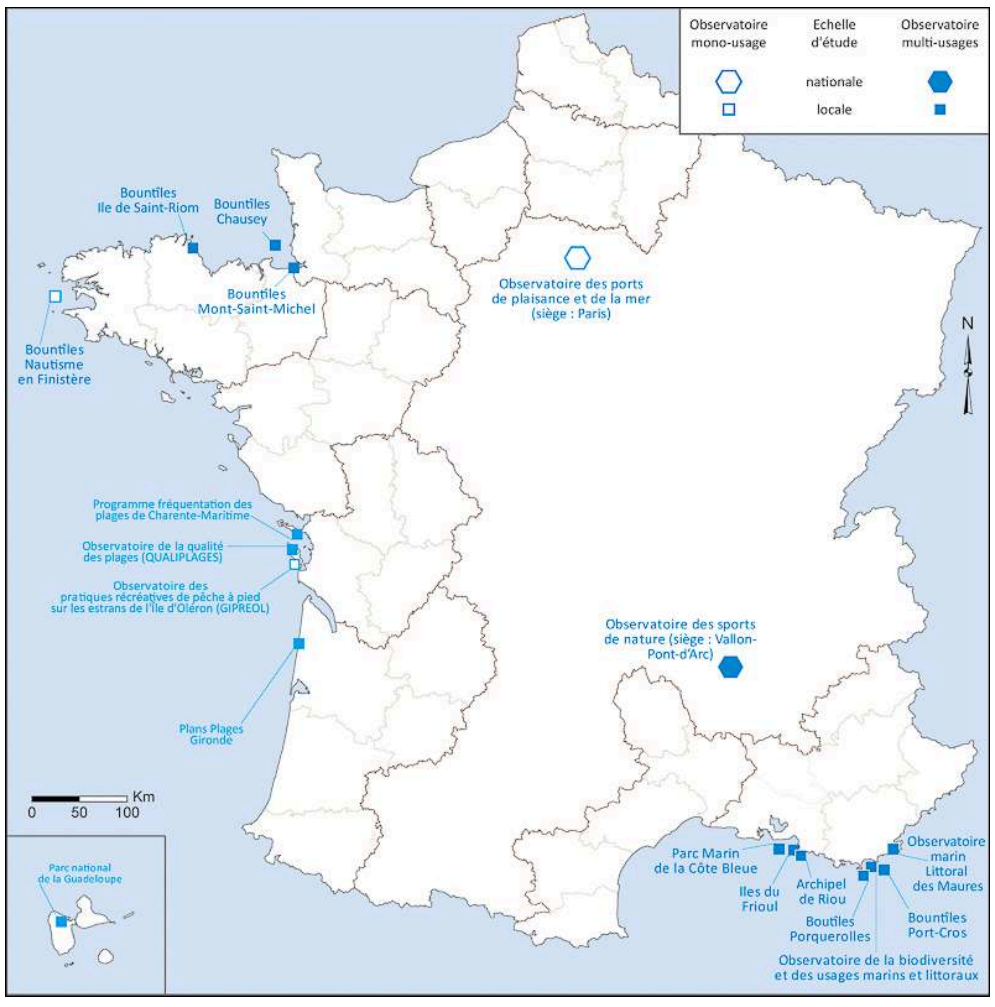

\section{Echelles de suivi de la fréquentation}

La prédominance des observatoires de la fréquentation implantés à l'échelle locale (15 sur 18) est peu surprenante dans la mesure où ces derniers produisent des données à la fois fines, précises et variées sur des aspects pointus de la fréquentation humaine, dans le but de répondre à des questionnements de gestion très concrets. C'est d'ailleurs pourquoi ces dispositifs sont essentiellement multi-usages (15 sur 18), les gestionnaires 
en ayant la charge souhaitant avoir une vision d'ensemble de la fréquentation sur leur site et non centrée uniquement sur une seule activité humaine ou une seule problématique.

Plus rares sont les observatoires de la fréquentation qui réalisent des suivis à petites échelles (3 sur 18). Mais cette notion de petite échelle mérite d'être relativisée. En effet, si les trois dispositifs considérés mènent effectivement une réflexion à l'échelle départementale ou nationale, tous se basent sur des données récoltées à grande échelle (c'est à dire au niveau local). C'est le cas, par exemple, de Bountîles Nautisme en Finistère qui vise à dénombrer et à spatialiser les mouillages déclarés et forains du département du Finistère, à quantifier et à qualifier la flotte de plaisance au mouillage, et ceci à partir de méthodes de suivi de terrain (prises de vues aériennes à partir de survols). Il en est de même pour l'observatoire des ports de plaisance et de la mer (Ministère de l'Environnement) qui vise à constituer une base de données précise des ports de plaisance français maritimes et fluviaux, et ceci à partir d'enquêtes administrées directement auprès des gestionnaires des ports considérés. Enfin, on peut citer l'observatoire des sports de nature (Ministère des Sports / Pôle Ressources National des Sports de Nature) qui a pour vocation prioritaire de diffuser des savoirfaire et de valoriser les bonnes pratiques et les actions innovantes afin de favoriser le développement maîtrisé des sports de nature sur le territoire.

\section{Objectifs des observatoires de la fréquentation}

37 La plupart des observatoires de la fréquentation (13 sur 18) présente la particularité d'avoir été créés pour répondre à un objectif commun : concilier l'accueil des publics avec des espaces naturels sensibles et souvent protégées. Ils sont considérés comme des outils visant à évaluer et suivre la pression de fréquentation et améliorer la gestion et la préservation des sites.

D'autres observatoires (4 sur 18) ont des objectifs plus généraux de développement durable : il s'agit de l'observatoire des ports de plaisance et de la mer (Ministère de l'Environnement), l'observatoire des Sports de nature, Bountîles Nautisme en Finistère ou encore Bountîles Saint-Riom. Ces derniers visent au développement harmonieux d'une ou plusieurs activités (la plaisance et ses infrastructures, les sports de nature) ou encore des usages insulaires.

Enfin, Bountîles Mont-Saint-Michel est un cas singulier puisqu'il vise avant tout à suivre l'évolution de la fréquentation touristique sur un site culturel majeur (inscrit au «Patrimoine Mondial » de l'UNESCO) dans le cadre du projet de rétablissement du caractère maritime du Mont-Saint-Michel. L'observatoire se présente, dans ce cas précis, comme un outil de connaissances sur lequel pourront s'appuyer les gestionnaires afin d'améliorer l'accueil des publics sur un site qui compte chaque année 2,5 millions de visiteurs.

\section{Analyse critique}

\section{Des observatoires peu nombreux et récemment mis en place}

Ces 18 observatoires recensés sont finalement peu nombreux à l'échelle nationale, au regard des centaines d'aires protégées françaises qui font pourtant l'objet d'enjeux de gestion liés à l'étude et au suivi de la fréquentation. Ces observatoires sont également 
insignifiants au regard de la superficie totale qu'ils couvrent au niveau national, quelques dizaines de milliers d'hectares seulement. Enfin, ces 18 dispositifs sont peu nombreux au regard de la multiplicité des observatoires environnementaux qui s'intéressent pourtant aux mêmes espaces naturels. Ainsi, alors que notre recherche n'était pas focalisée sur ces aspects, nous avons recensé indirectement, pas moins d'une cinquante de dispositifs visant à suivre et à qualifier les évolutions relatives aux milieux et/ou aux espèces. A titre d'exemple, nous pouvons citer les réseaux RNO (Réseau national d'observation de la qualité du milieu marin), REBENT (Réseau benthique), REMI (Réseau de contrôle microbiologique des zones de production conchylicoles), REPAMO (REseau PAthologie des Mollusques), REPHY (Réseau de suivi du PHYtoplancton et des phycotoxines), l'observatoires de l'état des populations de mammifères marins, l'observatoire des oiseaux marins, etc. Outre le fait d'être établis de longue date et parfois connus nationalement, ces observatoires environnementaux s'appuient sur des réseaux fortement structurés à la fois d'un point de vue institutionnel mais également géographique. Ce constat est bien évidemment lié à la longue implication des sciences de l'environnement (notamment de l'écologie marine et côtière) dans l'étude et la gestion des espaces naturels, et notamment des espaces naturels protégés. Inversement, on constate que les observatoires de la fréquentation sont des outils apparus très récemment sur ces mêmes espaces. Ainsi, sur les 18 observatoires de la fréquentation recensés, tous ont été créés à partir des années 2000 dont 12 après 2005.

\section{Des expériences souvent isolées, difficilement comparables, qui tendent à se structurer en réseau}

41 L'analyse croisée des observatoires de la fréquentation met en évidence une faible coordination, cohérence et interconnaissance (peu d'échanges d'expériences) entre les responsables des dispositifs recensés. La plupart de ces dispositifs sont le fruit d'expériences isolées, à l'initiative de structures gestionnaires, de bureaux d'étude, de laboratoires scientifiques ou encore d'organismes d'Etat ayant peu de relations entre eux.

Dans le détail, cette faible coordination doit cependant être relativisée. En effet, il est important de remarquer que sur les 18 observatoires de la fréquentation recensés, 10 d'entre eux font partie de réseaux qui leur sont propres :

- Les observatoires «Bountîles » forment un réseau comprenant 6 sites-ateliers. Créés et accompagnés par le laboratoire de géographie Géomer à Brest (UMR 6554 LETG CNRS), Bountîles (acronyme de «Base d'Observation des Usages Nautiques et Terrestres des Iles et des Littoraux ») est un outil permettant d'observer la fréquentation récréative et touristique des espaces marins, littoraux et insulaires, ainsi que ses impacts sur l'environnement (Le Berre, et al., 2010). Il fournit aux gestionnaires les éléments chiffrés, quantitatifs et qualitatifs, permettant de connaître les visiteurs et leurs pratiques et de mettre en place une politique de gestion de la fréquentation. Si chaque observatoire Bountîles est défini sur mesure en fonction du territoire étudié, en revanche, tous sont caractérisés par un fort degré d'intégration au sein du réseau (intégration en terme de démarche, de méthodes, de suivi, etc.)

- L'Observatoire des Pratiques de Tourisme et de Loisir est porté par le laboratoire de géographie LIENSs à la Rochelle (UMR 6250 CNRS) et se décline en trois « sousobservatoires » également fortement imbriqués : l'Observatoire des pratiques récréatives de 
pêche à pied sur les estrans de l'île d'Oléron (GIPREOL), le programme fréquentation des plages de Charente-Maritime et Qualiplages Oléron. «Créé en 2007, l'Observatoire des Pratiques de Tourisme et de Loisir vise à produire des connaissances sur les pratiques littorales et maritimes dans une perspective d'aide à la décision. Ses activités portent sur deux espaces concernés par la mise en place de Parcs Naturels Marins, l'archipel des Pertuis (îles de Ré et d'Oléron) sur la façade atlantique française et dans une moindre mesure le Département d'Outre-Mer de Mayotte » (http:// lienss.univ-larochelle.fr/ECOP.html).

- Enfin, le dispositif mis en œuvre par le Parc national de la Guadeloupe s'appuie en réalité sur une démarche plus globale de suivi de la fréquentation qui intègre l'ensemble des Parcs nationaux de France.

Malgré ces réseaux, l'ensemble des 18 observatoires de la fréquentation sur les espaces littoraux, marins et insulaires forme un tout encore largement hétérogène avec des niveaux d'intégration non comparables en termes de:

- Superficie : le plus petit observatoire couvre une superficie de 9 hectares (Bountîles SaintRiom) alors que le plus grand couvre une superficie de 21850 hectares (Parc national de Guadeloupe).

- Nombre de visiteurs : l'observatoire le moins fréquenté compte quelques dizaines de visiteurs par an seulement (Bountîles Saint-Riom), l'observatoire le plus fréquenté compte 2,5 millions de visiteurs par an (Bountîles Mont-Saint-Michel).

- Fréquence de suivi : certains observatoires comme celui du Parc national de la Guadeloupe ne réalisent que des suivis quinquennaux alors que d'autres réalisent des suivis annuels voire mensuels ou quotidiens.

-Validation scientifique : on distingue les observatoires qui ont été créés par des organismes de recherche spécialisés sur l'étude de fréquentation, les observatoires dont le protocole de suivi a été validé par le comité scientifique d'une aire protégée et les observatoires non validés scientifiquement.

- Méthodes, de protocoles de suivi et de collecte des données : si ces derniers sont variables en fonction de chaque site (littoral, insulaire ou marin), ils le sont aussi entre sites pourtant de même nature. Il n'existe pas d'harmonisation dans le format et le contenu des outils, ce qui n'autorise pas des comparaisons entre sites et des analyses globales à petite échelle. De la même façon, on constate que le nombre et la qualité des indicateurs varient d'un site à l'autre. Si certains observatoires se contentent de deux ou trois indicateurs (par exemple : le traitement des données de compteurs automatiques couplé à la réalisation de questionnaires), d'autres s'appuient sur plus d'une vingtaine d'indicateurs pour suivre la fréquentation.

- Bancarisation des données : il s'agit d'une question complexe mais cruciale pour tout organisme qui souhaite suivre des évolutions sociales dans le temps. Les réponses apportées sont là encore hétérogènes : stockage papier, tableur Excel, logiciels spécialisés (d'économétrie ou de sociologie) ou encore base de données sophistiquée avec interface interactive.

- Modes de restitution des résultats : si certains observatoires parviennent effectivement à mettre en valeur leurs données à travers des rapports, des fiches synthétiques, des atlas, des publications scientifiques, d'autres peinent à traiter des données qui s'accumulent progressivement et parfois inutilement, faute de moyens humains et/financiers mais aussi faute de savoir-faire...

- Opérationnalité mais aussi de crédibilité : certains observatoires ont gagné leur légitimité car ils ont su répondre précisément aux questionnements et aux besoins initiaux de leurs 
gestionnaires. En revanche, d'autres ne parviennent toujours pas à identifier les enjeux de la fréquentation locaux qui sous-tendent pourtant leur existence.

\section{Une faible valorisation scientifique et technique}

Enfin, l'analyse des observatoires de la fréquentation met en évidence une littérature globalement pauvre. Celle-ci est caractérisée par la faiblesse des publications de qualité au profit de synthèses et de rapports internes détaillant de façon plus ou moins précises (voire obscure) les méthodologies employées (protocoles, collecte et archivage des données, restitution et utilisation des données) ainsi que les résultats des différentes campagnes de suivi réalisées. La faiblesse du nombre des références méthodologiques et généralistes ayant pour objectif d'aider à la mise en œuvre et à l'accompagnement d'un observatoire de la fréquentation est particulièrement criante.

\section{D'autres expériences d'observatoires de la fréquentation en France et à l'étranger}

\section{Les observatoires terrestres en France}

Si la recherche bibliographique réalisée n'était pas ciblée sur les observatoires de la fréquentation strictement terrestres (espaces montagnards, fluviaux, forestiers, etc.), elle nous a cependant permis de recenser quelques expériences majeures en France. Celles-ci sont au nombre de 11 (carte 2).

Carte 2 - Quelques observatoires opérationnels de la fréquentation dans les espaces terrestres français

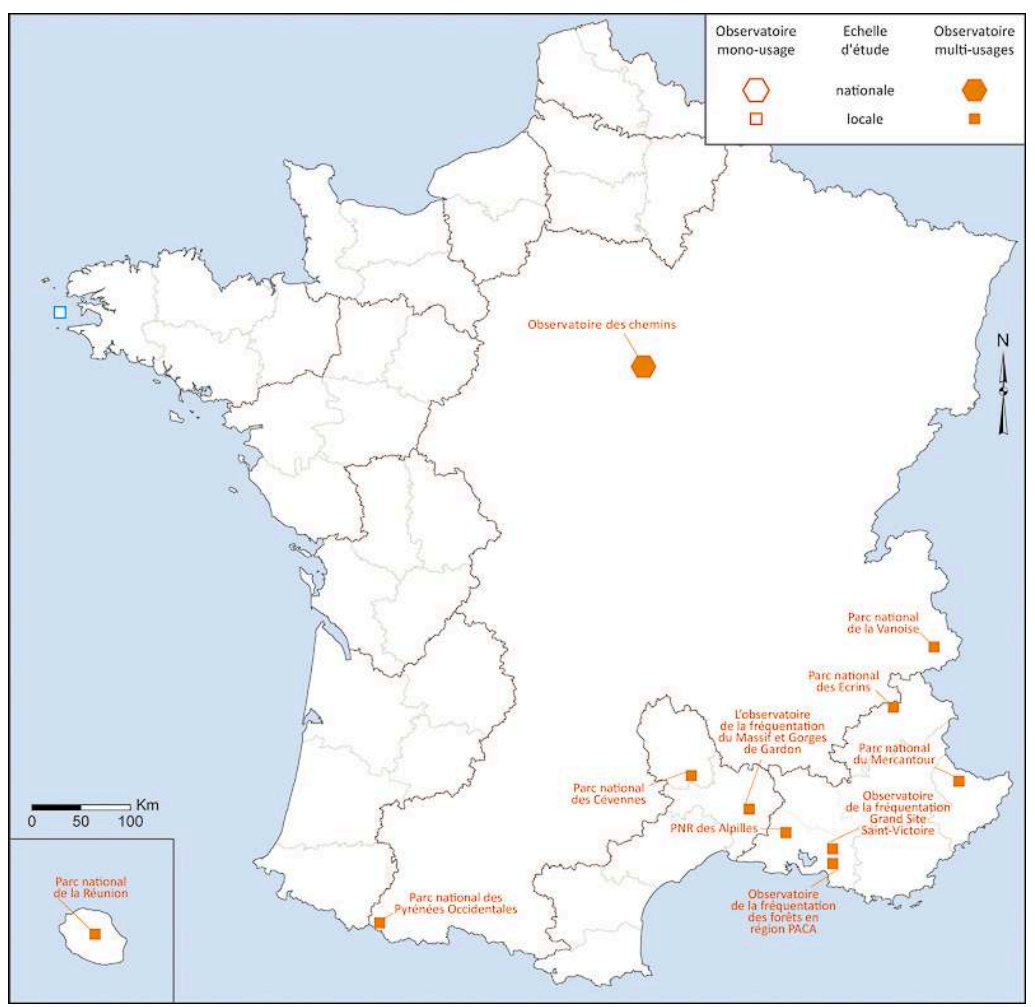

EchoGéo, 19 | 2012 

constituent les expériences terrestres les plus significatives. Les Parcs nationaux font partie des premiers organismes ayant réalisé des études de fréquentation. Ils sont également les premiers à avoir réfléchi et mis en œuvre des systèmes de suivi communs à l'ensemble du réseau. Dès 1996, les Parcs de la Vanoise, des Écrins, du Mercantour et des Pyrénées adoptent la même démarche et les mêmes protocoles d'étude (Parc national de la Vanoise, 1996). Ils seront suivis en 2006 par le Parc de la Guadeloupe, puis en 2011 par les Parcs de la Réunion et des Cévennes. d'informations nouvelles par rapport à l'analyse déjà réalisée sur les espaces littoraux, marins et insulaires. En revanche, celle-ci vient confirmer et renforcer le constat fait précédemment :

- un faible nombre des dispositifs terrestres sur l'ensemble du territoire français mais également des superficies de territoires couvertes réduites (malgré la taille conséquente des Parcs nationaux),

- des expériences globalement isolées caractérisées par l'absence d'harmonisation nationale,

- des niveaux d'intégration non comparables en termes de fréquence de suivi, de méthodes et de protocoles de collecte de terrain, de nombre et de qualité d'indicateurs, de bancarisation, etc.

- une bibliographie pauvre, notamment en ce qui concerne les références méthodologiques et généralistes.

\section{Les observatoires de la fréquentation à l'étranger}

Une recherche bibliographique spécifique (plus simple et plus rapide), à partir de motsclés en langue anglaise, a également été réalisée afin d'explorer la littérature internationale. Deux bases de données seulement ont été utilisées (Google et Google Scholar) ainsi que quelques combinaisons de mots-clés. Ces derniers sont cependant unanimement utilisés par les spécialistes : "visitor monitoring " (équivalent de "étude de fréquentation), "visitor monitoring system" (équivalent d'observatoire de la fréquentation), « protected area », « natural area ».

Il apparaît, à ce stade de la recherche, que la littérature internationale relative aux observatoires de la fréquentation humaine est peu abondante. Ce constat révèle que la situation décrite en France est comparable à celle connue à l'étranger, à quelques exceptions mises à part. Certains spécialistes, tels que A. Muhar, et al.(2002) mettent, par exemple, en évidence que dans la plupart des pays européens, "les suivis de la fréquentation humaine sont rares et lorsqu'ils existent sont le plus souvent organisés dans une base de données ad hoc sans réelle planification ». G. Cessford et R. Burns (2008) confirment que "la recherche sur les usages récréatifs et touristiques ne fait pas l'objet d'une tradition de longue date et a d'ailleurs souvent été négligée en comparaison des études biologiques et ceci alors que les connaissances sur les visiteurs sont un besoin élémentaire pour n'importe quelle gestion des services, des infrastructures et des impacts » dans les espaces de nature.

Cependant la situation évolue peu à peu si l'on tient compte d'un certain nombre d'initiatives internationales (et notamment européennes) visant à mettre en œuvre des dispositifs de suivi de la fréquentation pérennes dans le temps. C'est le cas de pays comme le Danemark qui cherche à pérenniser le suivi de la fréquentation humaine dans ses forêts à partir d'enquêtes postales réalisées à l'échelle nationale, d'enquêtes 
locales et de comptages de terrain (Skov-Petersen et Søndergaard Jensen, 2004). C'est le cas également de l'Angleterre, où l'agence gouvernementale "English Nature " (gestionnaire de plus de 200 réserves nationales) envisage un système de comptage harmonisé à partir de la méthode des compteurs automatiques pour l'ensemble de ses aires protégées (Melville et Ruohonen, 2004). Enfin, citons des pays comme la Finlande, la Belgique, l'Allemagne, la Norvège ou encore l'Estonie qui développent peu à peu des approches standardisées sur leur territoire, à l'échelle nationale, régionale ou locale (Sievänen et al., 2008).

51 Si les réalisations européennes restent encore largement au stade d'expérimentation, d'élaboration et de structuration, en revanche, certains projets étrangers ont atteint une maturation certaine avec des dispositifs de suivi de la fréquentation établis de longue date. Ces pays expérimentés sont très peu nombreux : Australie, Canada, ÉtatsUnis, Nouvelle-Zélande (tableau 6). Les observatoires développés dans ces pays s'appuient sur des méthodes fortement standardisées permettant de suivre la fréquentation dans ses aspects aussi bien quantitatifs et qualitatifs que comportementaux. Les données collectées autorisent aujourd'hui la production d'informations sur des tendances horaires, journalières et/ou saisonnières à l'échelle locale tout en permettant des comparaisons et des analyses à l'échelle nationale.

Tableau 6 - Quelques dispositifs de la fréquentation à l'étranger

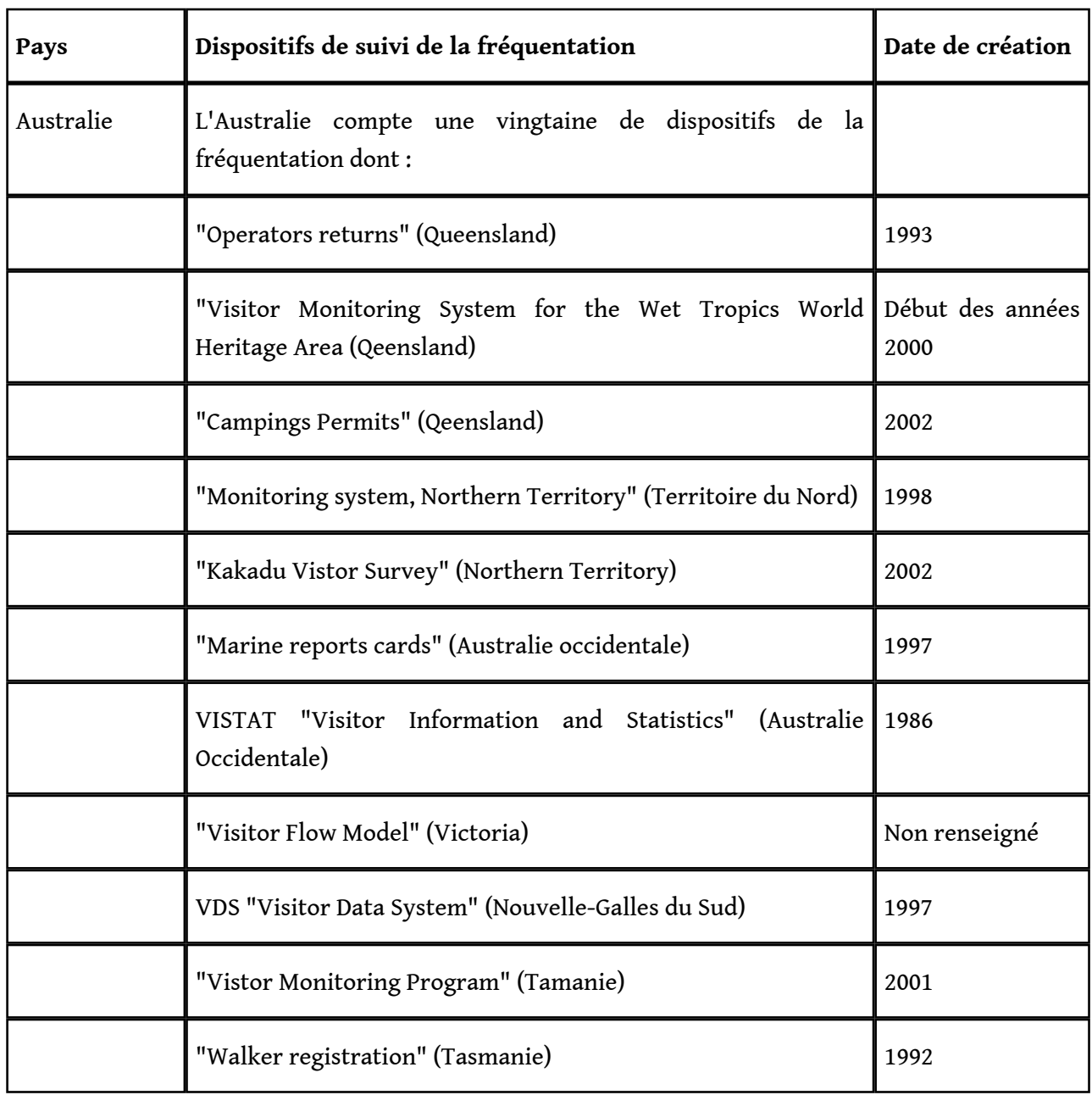




\begin{tabular}{|l|l|l|}
\hline & Etc. & \\
\hline Canada & "Visitor Activites Management Process" & $\begin{array}{l}\text { Début des années } \\
1990\end{array}$ \\
\hline États-Unis & "National Visitor Use Monitoring" & 1998 \\
\hline $\begin{array}{l}\text { Nouvelle- } \\
\text { Zélande }\end{array}$ & "The Visitor Asset Management System" & 1998 \\
\hline
\end{tabular}

Source : Wardell et Moore, 2005; Brown et al., 2006

53 Aux Etats-Unis, le National Visitor Use Monitoring (NVUM) fait partie des dispositifs de suivi de la fréquentation les plus élaborés au monde. Celui-ci vise à estimer le nombre total et les caractéristiques des visiteurs pour l'ensemble du réseau de forêts fédérales du pays qui couvrent $769000 \mathrm{~km}^{2}$ (the "National Forest System ») (USDA Forest Service, 2010). Ce dernier a été imaginé en 1998 par une équipe constituée de chercheurs spécialisés et de gestionnaires. Il s'appuie sur des méthodes d'étude mises au point dès les années 1970 par l'USDA Forest Service, et améliorées au cours des années 1990. Après avoir été testé pour la première fois entre 2000 et 2003 puis révisé et amélioré pendant l'année 2004, l'ensemble du système devient opérationnel à partir de 2005 (English et al., 2001). Le National Visitor Use Monitoring a notamment permis d'estimer à 1184000 la moyenne annuelle de visiteurs dans les forêts américaines sur la période 2005-2010 (USDA Forest Service, 2011).

\section{Conclusion}

54 Cette recherche sur l'état de l'art en matière d'observation et de suivi des usages liés aux fréquentations des sites marins, littoraux et insulaires a permis de mettre en évidence un certain nombre de conclusions tout à fait éclairantes sur le faible intérêt porté à ce sujet par la communauté scientifique appartenant au domaine des sciences humaines et sociales.

On retiendra en premier lieu que les équipes s'intéressant à ces questions sont peu nombreuses et que cette thématique, pourtant d'actualité du fait de l'accroissement des activités maritimes et de la mise en place récente d'un grand nombre de structures de conservation et de gestion, comme les parcs naturels marins, reste peu étudiée par les chercheurs. On notera également que les réflexions en cours sont le fait de petites équipes, voire de chercheurs isolés travaillant le plus souvent à l'échelle d'un ou de plusieurs sites de faibles superficies. Ce constat explique que les connaissances apparaissent fragmentées dans l'espace et cloisonnées au plan disciplinaire. Le petit nombre d'observatoires proposant de réels suivis de la fréquentation est également une conséquence de la faible mobilisation des chercheurs en SHS sur ces questions. Enfin, l'absence de standardisation des méthodes d'étude des usages récréatifs et touristiques dans les aires protégées limite considérablement les possibilités de comparaison entre sites et toute analyse sérieuse à l'échelle nationale.

Cette réflexion sur l'état de l'art propose un point structuré, organisé et hiérarchisé de l'information scientifique disponible au niveau national, mais aussi international. Il 
convient cependant de rappeler certaines limites inhérentes à ce type d'exercice et rendues inévitables du fait de l'ampleur du sujet. Ainsi, certains aspects n'ont pas été pris en compte, comme le suivi des activités économiques portuaires, ou les flux des navires de commerce. De même, certaines difficultés d'analyse ont pu se présenter du fait que plusieurs méthodes d'étude et de suivi de la fréquentation sont développées par des corpus disciplinaires autres que ceux des auteurs ayant rédigé le présent article (des géographes). Par conséquent, si cet état de l'art pose et organise les premières bases indispensables d'une réflexion méthodologique, il conviendra de le compléter, sur certains aspects, par l'apport d'autres approches disciplinaires.

La complexité des systèmes de fréquentation oblige désormais à mutualiser les compétences d'équipes et disciplines différentes mais complémentaires afin de mieux décrypter et comprendre les situations actuelles. Il semble aujourd'hui plus que nécessaire d'établir des programmes scientifiques communs et interdisciplinaires permettant de faciliter les partenariats et les synergies entre chercheurs. C'est à cette condition que les connaissances se développeront et aboutiront à la réalisation d'outils d'observations et de suivis solides et indispensables, tant pour les scientifiques que pour les gestionnaires. Un projet de Groupement d'Intérêt Scientifique (GIS) Fréquentation, Usages et Gouvernance des aires marines protégées est actuellement en cours de réflexion au sein de l'Université de Bretagne Occidentale en relation avec l'Agence des Aires Marines Protégées. Ce GIS sera probablement la structure la plus à même de relever un tel défi et de faire avancer la recherche scientifique dans ces domaines.

\section{BIBLIOGRAPHIE}

Audouit C., (2009). L'étude de la fréquentation et de ses impacts sur le littoral languedocien. Thèse de Géographie, Université de Montpellier III, Montpellier, 557 p.

Baron-Yellès N., 1997. Espaces protégés et tourisme de nature sur le littoral atlantique français. Stratégies et enjeux. Thèse de Géographie, Université Paris 1 Sorbonne, 370 p.

Brigand L., Le Berre S., 2007. Joint construction and appropriation of indicators by the users, the managers and the scientists: the example of the touristic frequentation observatory of Port-Cros and Porquerolles. International Journal of Sustainable Development, 10, 1 et 2, p. 139-160.

Brigand L., Le Berre S., 2009. Tourisme et environnement dans les espaces protégés littoraux et insulaires. Evaluation et proposition d'outils méthodologiques pour l'observation, la gestion, la prévision et la concertation. Brest, rapport laboratoire Géomer UMR 6554 LETG, UBO, programme national de recherche Liteau, MEEDDAT, $102 \mathrm{p}$.

Brown, G. et al., 2006. Managing Australia's protected areas: review of visitor management models, frameworks and processes. CRC for Sustainable Tourism, Gold Coast, Qld., 98 p.

Cessford G., Burns R., 2008. Monitoring visitor numbers in New Zealand national parks and protected areas. A literature review and development summary. Science \& Technical Publishing, Department of Conservation, New Zealand, $47 \mathrm{p}$. 
Chlous-Ducharme F. et al., 2008. Modélisation et jeu de rôles sur l'île d'Ouessant : questions de sociologie. Socio-logos, $\mathrm{n}^{\circ} 3$, http://sociologos.revues.org/document2112.html

David, G. et al., 2006. Les concordances territoriales au cœur de la gestion intégrée des zones côtières : l'exemple de la Réunion. Actes du colloque international « Interactions Nature-Société, analyse et modèles ", La Baule, 2006, 6 p.

Duhamel P. et al., 2002 . Tourisme 1. Lieux communs. Paris, Editions Belin, Collection Mappemonde, $320 \mathrm{p}$.

Duvat V. (dir.), Mossot G., 2011. Guide de qualité des plages de l'île d'Oléron, QUALIPLAGES, 330 p.

English D. B. K., Kocis S. M. et al., 2001. Forest Service National Visitor Use Monitoring Process: Research Method Documentation.USDA, Forest Service, $39 \mathrm{p}$.

Evrard B., 2010. La côte d'Albatre comme terrain de jeux? Aménagements, activités physiques et paysages vécus. Thèse de STAPS, Université de Rouen, $383 \mathrm{p}$.

Fleury P.-G., Cadet C., 2010. Analyse des données de pêches à pied traditionnelles et de chasse sousmarine, pratiquées en 2008 et 2009 dans la Réserve Naturelle Marine de La Réunion. IFREMER, 29 p.

Goeldner-Gianella L., Humain-Lamoure A.-L., 2010. Les enquêtes par questionnaire en géographie de l'environnement. L'Espace Géographique, 4, p. 325-344.

Jollit I., 2010. Spatialisation des activités humaines et aide à la décision pour une gestion durable des écosystèmes coralliens. La pêche plaisancière dans le lagon sud-ouest de la Nouvelle-Calédonie. Thèse de doctorat en Géographie, Université de la Nouvelle-Calédonie, 558 p.

Le Berre S., 2008. Les observatoires de la fréquentation, outils d'aide à la gestion des îles et des littoraux. Brest, thèse de Géographie, Géomer LETG UMR 6554 CNRS, UBO, 745 p.

Le Berre S. et al., 2010. Bountîles. Un outil pour une meilleure gestion de la fréquentation. A PortCros, l'observatoire est aussi un outil de concertation. Espaces, 278, p. 17-21.

Le Corre, N. et al., 2011. Dispositifs de suivi de la fréquentation des espaces marins, littoraux et insulaires et de ses retombées socio-économiques : état de l'art. Brest, rapport Géomer LETG, UMR 6554 et UMR M101 Amure, Université de Bretagne Occidentale, Agence des Aires Marines Protégées, 150 p.

Melville S., Ruohonen J., 2004. The development of a remote-download system for visitor counting. Working Papers of the Finnish Forest Research Institute 2, p. 38-44.

Muhar A. et al., 2002. Methods for Visitor Monitoring in Recreational and Protected Areas: An Overview. Monitoring and Management of Visitor Flows in Recreational and Protected Areas. Conference Proceedings, $6 \mathrm{p}$.

Musard O., 2003. Les pratiques subaquatiques au sein des aires marines protégées de Méditerranée française : entre paysages sous-marins, représentations et impacts. Contribution au développement d'une géographie relative aux territoires sous-marin. Thèse de géographie (sous la dir. de G. Richez), Université d'Aix-Marseille 1, Maison Méditerranéenne des Sciences de l'Homme, Aix-enProvence, $449 \mathrm{p}$.

Parc national de la Vanoise, 1996. Spécial «étude de fréquentation» 1996. Parc national de la Vanoise, $10 \mathrm{p}$.

Parrain C., 2010. Territorialisation des espaces océaniques hauturiers. L'apport de la navigation à voile dans l'Océan Atlantique. Thèse de Géographie, Université de la Rochelle, s.p. 
Peuziat I., 2005. Plaisance et environnement. Pratiques, représentations et impacts de la fréquentation nautique de loisir dans les espaces insulaires. Le cas de l'archipel de Glénan (France). Brest, thèse de Géographie, Université de Bretagne Occidentale, $344 \mathrm{p}$.

Picon B., 1987. Les conflits d'usage sur le littoral camarguais : protection de la nature et pratiques sociales. Norois, 34, 133-135, p. 73-80.

Richez G. 1995. Réserve naturelle des Iles Lavezzi : La fréquentation touristique et récréative de l'île Lavezzu durant l'été 1994 et évolution 1991-1994. Travaux scientifiques du Parc naturel régional et des réserves naturelles de Corse, 55, p. 45-92.

Sabinot C., 2008. Dynamique des savoirs et des savoir-faire dans un contexte pluriculturel. Étude comparative des activités littorales au Gabon. Thèse du Muséum National d'Histoire Naturelle, USM104 - CNRS, UMR5145 - Université Paris Diderot - Paris 7, 438 p.

Sievänen, T. et al., 2008. Forest Recreation Monitoring: a European Perspective. Working Papers of the Finnish Forest Research Institute, Finish Forest Research Institute, Finlande, 245 p.

Skov-Petersen H., Søndergaard Jensen, F., 2004. Assessing recreation in the Danish nature - present experiences, towards a future monitoring system. Working Papers of the Finnish Forest Research Institute 2, p. 58-65.

Thomassin A., 2011. "Des réserves sous réserve ». Acceptation sociale des Aires Marines Protégées. L'exemple de la région sud-ouest de l'océan indien. Thèse de Géographie, Université de la Réunion, UMR ESPACE-DEV - IRD, Réunion, 566 p.

Trouillet B., 2006. La mer-territoire ou la banalisation de l'espace marin (golfe de Gascogne). Mappemonde, 4, 84, Montpellier, $17 \mathrm{p}$.

Vacher, L. (dir.) et al., 2011. Méthodologie traitements Atlas de la fréquentation des plages de CharenteMaritime. La Rochelle, UMR LIENSs, ECOP-CelluleGéomatique, 53 p.

USDA Forest Service, 2011. Visitor use report. National Visitor Use Monitoring. Data collected FY 2005. USDA Forest Service, $51 \mathrm{p}$.

Wardell M., Moore S., 2004. Collection, storage and application of visitor use data in protected areas. Guiding principles and case studies. CRC for Sustainable Tourism, Gold Coast, Qld., 104 p.

\section{NOTES}

1. Ces bases de données bibliographiques sont les suivantes: Academic Search Premier, Archimer, Asfa, Avano, Cairn, Catalogue La Pérouse, DOAJ, Google, Google Scholar, HAL Jstor, Pascal, Persée, Revue.Org, Science Direct, Scirus, SpringerLink, Thèse En Ligne, Web of Science, Wiley-Blackwell.

2. Même au sein de ces équipes, le nombre de chercheurs est souvent très faible, d'où la fragilité de la continuité de la recherche dans certains laboratoires de recherche. 


\section{RÉSUMÉS}

L'essor du tourisme de nature génère un attrait grandissant pour les espaces naturels protégés littoraux. Cet engouement pose de multiples enjeux liés à la gestion de la fréquentation et à l'acquisition de connaissances sur ces espaces souvent sensibles. Les suivis environnementaux apparaissent comme des outils aujourd'hui largement développés et aboutis. En revanche, la caractérisation des activités humaines répond à une préoccupation plus récente et soulève de nombreuses questions scientifiques. Cet article propose, à travers une revue de littérature menée sous l'angle des Sciences Humaines et Sociales, un recensement et une analyse des méthodes d'étude des usages récréatifs et touristiques sur les espaces littoraux, marins et insulaires. Il s'agit également de montrer comment ces outils sont exploités en France afin d'alimenter des observatoires de la fréquentation. Caractérisée par des approches cloisonnées, la recherche française gagnerait à se structurer en mutualisant les compétences d'équipes et de disciplines différentes mais complémentaires.

The coastal natural areas are getting more and more attractive because of the tourist growth. This increasing interest leads to several issues linked to visitor management impacts. Today, environmental monitoring seems to be widely developed and satisfactory. On the other hand, visitor monitoring is a recent preoccupation witch raises several scientific questions. Through an important literature review led by the social sciences, this article proposes an inventory and an analysis of the methods to obtain visitor data on the coastal, the sea and insular natural areas. Then, it shows how these methods are used in France in order to develop visitor monitoring system. The French research is characterized by various but compartmentalized approaches. It could make considerable progress and improvements by mixing the team skills and the different but complementary disciplines.

\section{INDEX}

Mots-clés : aire marine, fréquentation, île, littoral, méthode, observatoire, recensement

Keywords : France, inventory, marine natural area, method, review, visitor monitoring system Thèmes : Sur le Champ - Sur le Terrain

\section{AUTEURS}

\section{NICOLAS LE CORRE}

Nicolas Le Corre, nicolas.lecorre@univ-brest.fr, est docteur en géographie, chercheur associé au laboratoire Géomer (LETG, UMR 6554 du CNRS). Il a publié récemment :

- Le Corre N., Brigand L., et al., 2011. Dérangement de l'avifaune : croiser étude de fréquentation et suivi naturaliste dans le cadre d'un outil d'aide à la gestion. In : Triplet, P. (sous la dir.), 2011. Manuel de gestion des oiseaux et de leurs habitats dans les écosystèmes estuariens et littoraux, 32 p, à paraître.

\section{SOLENN LE BERRE}

Solenn Le Berre, solenn.leberre@univ-brest.fr, est docteur en géographie, chercheur associé au laboratoire Géomer (LETG, UMR 6554 du CNRS). Elle a publié récemment :

- Le Berre S. et Brigand L., 2011. La pêche à pied dans l'archipel de Chausey (France). Contribution à une 
analyse des politiques de gestion durable de l'estran à travers la prise en compte des activités récréatives. Actes du colloques Insularité et développement durable, 24-27 novembre 2009, Saint-Denis de la Réunion (France), Editions de l'IRD, à paraître.

\section{LOUIS BRIGAND}

Louis Brigand, louis.Brigand@univ-brest.fr, est Professeur de géographie, directeur du laboratoire Géomer (LETG, UMR 6554 du CNRS). Il a publié récemment :

- Le Berre S., Brigand L., Morio A., 2010. Bountîles, un outil pour une meilleure gestion de la fréquentation. Espace Insularité et Tourisme, 278, p. 17-21.

\section{INGRID PEUZIAT}

Ingrid Peuziat, ingrid.peuziat@univ-brest.fr, est Maître de conférences à l'Université de Bretagne occidentale et membre du laboratoire Géomer (LETG, UMR 6554 du CNRS). Elle a publié récemment :

- Peuziat I., 2004. Plaisanciers en quête d'espaces naturels et de tranquillité : illusion ou réalité ? Le cas de l'archipel de Glénan (France). Norois, 4, 1993, (en ligne). 